\title{
Modeling of Self-Vibratory Drilling Head-Spindle System for Predictions of Bearings Lifespan
}

\author{
F. Forestier, ${ }^{1}$ V. Gagnol, ${ }^{2}$ P. Ray, ${ }^{2}$ and H. Paris ${ }^{3}$ \\ ${ }^{1}$ LaMI Laboratory, Clermont Université, UBP, EA 3867, BP2235, 03101 Montlucon, France \\ ${ }^{2}$ LaMI Laboratory, Clermont Université, IFMA, EA 3867, BP10448, 63000 Clermont-Ferrand, France \\ ${ }^{3}$ G-SCOP Laboratory, G-SCOP, 46 Avenue Félix Viallet, 38031 Grenoble, France
}

Correspondence should be addressed to F. Forestier, fabien.forestier@moniut.univ-bpclermont.fr

Received 13 January 2011; Accepted 7 June 2011

Academic Editor: Atma Sahu

Copyright (C) 2011 F. Forestier et al. This is an open access article distributed under the Creative Commons Attribution License, which permits unrestricted use, distribution, and reproduction in any medium, provided the original work is properly cited.

\begin{abstract}
The machining of deep holes is limited due to inadequate chip evacuation, which induces tool breakage. To limit this drawback, retreat cycles and lubrication are used. An alternative response to the evacuation problem is based on high-speed vibratory drilling. A specific tool holder induces axial self-maintained vibration of the drill, which enables the chips to be split. The chips are thus of a small size and can be evacuated. To anticipate the potential risk of decreased spindle lifespan associated with these vibrations, a model of the behavior of the system (spindle_-self-vibrating drilling head-tool) is elaborated. In order to assess the dynamic behavior of the system, this study develops a rotor-based finite element model, integrated with the modelling of component interfaces. The current results indicate that the simulations are consistent with the experimental measurements. The influence of spindle speed and feed rate on bearing lifespan is highlighted.
\end{abstract}

\section{Introduction}

High-speed vibratory drilling allows chips to be split thanks to self-maintained vibration during cutting [1]. When these vibrations have a magnitude greater than the advance per tooth, the drill continuously enters and exits the material, which allows the fragmentation of the chips, as shown by Tichkiewitch et al. [2]. The chips are small and can thus be removed easily without retreat cycles or lubrication. Highspeed vibratory drilling enables productivity to increase by a factor of three compared to traditional techniques. Currently, high-speed vibratory drilling is the only process capable of producing deep holes using high-speed machining centers, without lubricants, and with high productivity [3].

However, the excitation generated by the cutting process can also be a source of damage to the self-vibrating drilling head and the spindle. So the industrialization of high-speed vibratory drilling requires the effects of vibration on the machine, and more particularly on the spindle, to be predicted. For this purpose, a model of the dynamic behavior of the system (self-vibrating drilling head spindle) is elaborated from a realistic assembly of the components, in order to predict spindle bearing lifespan.
The SVDH is composed of an axial vibrating system, consisting of the SVDH vibrating subsystem, mounted on a specific HSK 63 taper, and called the SVDH body. The SVDHvibrating subsystem is composed of the vibrating parts of the drill holder. The SVDH body guides the axial vibrating subsystem through a ball retainer and a classic HSK63 taper connexion with the spindle head. The self-excited vibrations must be tuned and controlled in order to have a magnitude greater than the advance per tooth. Mathematical models of the SVDH dynamics appearing in most of previous works lead to a one-dimensional linear or nonlinear model in the axial vibration direction governed by the mass, damping, and stiffness of the considered system [3]. In these studies, damping, which plays an important role in SVDH dynamic behaviour, is estimated but not experimentally identified. Moreover, the spindle behaviour and the interfaces between the spindle and the SVDH are assumed to be rigid and have not been taken into account. However, many works have showed that tool point dynamics can be significantly influenced by spindle dynamics, as well as the spindle-holdertool interfaces. This creates a demand for predictive knowledge models that are capable of investigating the influence of cutting conditions on high-speed spindle-SVDH system. 
Many authors have investigated the dynamic behaviour of machine tool spindle-bearing systems, both analytically and experimentally. They show that spindle dynamics are influenced by a large number of factors, including holder characteristics [4], spindle shaft geometry and drawbar force $[5,6]$, and the stiffness and damping provided by the bearings [7]. Most of these factors are independent of spindle speed, contrary to bearing properties $[8,9]$ and the spindle rotor dynamics, which change according to spindle speed. Such rotating systems have been successfully modelled through rotor-dynamics studies $[10,11]$. In previous works $[12,13]$, a dynamic high-speed spindle-bearing system model based on rotor dynamics prediction was presented. Element kinematics were formulated in a corotational coordinate frame and enabled a special rotor beam element to be developed. Model results showed that spindle dynamics are influenced by the gyroscopic coupling and the spin softening of the rotating shaft due to high rotation speeds.

The literature on the modelling and analysis of spindles shows that the tool tip FRF is also greatly influenced by the contact dynamics of the spindle-holder-tool interfaces. The flexibility of the afore-mentioned interfaces can dominate the dynamics of the spindle. The tool tip FRF is usually obtained using experimental measurements, which require significant testing time to take into account the large number of spindle, holder, and tool combinations. Thus, semi-analytical approaches have been proposed to minimise experimental approaches. Erturk et al. [14] use a receptance coupling (RC) and structural modification method to connect the spindle shaft and the tool holder. Schmitz et al. [15] model the spindle holder experimentally and couple it with an analytical model of the overhang portion of the tool. Recent approaches consider distributed springs and dampers between the tool and the holder along the interface contact. $[15,16]$. Contact stiffness and damping values alter the frequencies and peak values respectively of dominant tool tip vibration modes. The fast and accurate identification of contact dynamics in spindle-tool assemblies has become an important issue. Ren and Beards [17], Schmitz et al. [15], and Movahhedy and Gerami [18] treat identification as a nonlinear optimisation problem. Movahhedy and Gerami [18] uses a genetic algorithm to find the global minimum of the optimisation function

$$
|g|=\left|\left\{\begin{array}{c}
\operatorname{Re}\left(g_{p}-g_{m}\right) \\
\operatorname{Im}\left(g_{p}-g_{m}\right)
\end{array}\right\}\right|,
$$

where $g_{p}$ and $g_{m}$ are, respectively, predicted and measured receptances of the assembly. Ozsahin et al. [19] present an original identification procedure based on experimental measurements. In their work, the elastic RC equations allow the stiffness and damping parameters of the spindle-tool assemblies to be obtained in closed-form expressions. In order to predict the drill dynamics and the adequate cutting conditions that lead to controlled self-excited vibrations, an accurate comprehensive dynamic model of the cutting process and spindle-SVDH dynamics is required.

In this paper, a hybrid model based on numerical and experimental approaches of the dynamic behavior of the system is proposed. This finite element model takes into account rotor dynamics effects, bearing stiffness and the real behavior of interfaces between different system components. The simulation allows achieving the best possible cutting parameters without damaging the bearings of the spindle.

In the second section, the spindle-SVDH rotor dynamics model is presented. A special rotor-beam element is implemented. The rolling bearing stiffness matrices are calculated around a static function point on the basis of Lim and Singh's [20] formulation and then integrated into the global finite element model. The identification of contact dynamics in tool-SVDH-spindle assemblies is carried out using the RC method on the basis of experimental substructure characterisation. The identified models are then integrated into the global spindle-SVDH-tool model. Finally, numerical and experimental tool tip FRF, in radial and axial directions, is compared in order to validate the global assembled model.

Section 3 is dedicated to the definition of optimal cutting conditions with respect to industrial objectives. The studied industrial context requires the maximum material cutting rate and a rational use of the tool-SVDH-spindle set in order to guarantee adequate rolling bearing lifespan. As a result, a recommendation for the use of a spindle-SVDH-tool set which respects the defined zone of interest, combining reli ability and productivity constraints, is proposed.

Finally, conclusions are presented.

\section{Model Building}

The vibratory drilling system is composed of a SVDH body clamped to the spindle by a standard HSK63A tool-holder interface. A SVDH-vibrating subsystem is jointed to the SVDH body with a specific spring and axially guided by a ball retainer. Finally, a long drill is held in the SVDH-vibrating subsystem with a standard ER25 collet chuck. The spindle has four angular bearings in overall back-to-back configuration (Figure 1).

The spindle-SVDH-tool finite element model is restricted to the rotating system composed of the spindle shaft, the SVDH and the drill. An experimental modal identification procedure was carried out on the different spindle substructures and showed that spindle behaviour can be restricted to rotating structure behaviour [12]. The interfaces represented by the HSK63 taper, spring and ball retainer, and collet chuck were taken into account in the model. The CNC milling machine structure was assumed to be infinitely rigid compared to the other parts of the system.

The numerical model of the spindle SVDH tool is based on the integration of the rotating system's finite element model (FEM), the rolling bearing model, and the interface model. Figure 2 summarises the various stages of model development. The system substructures were modelled through rotor-dynamics formulations. A readjustment procedure was carried out on undefined FEM material properties in order to fit model results to experimental ones. The receptance coupling method was used to identify the dynamic parameters of the system's interfaces. Once each structural subsystem model was validated, the identified interface 

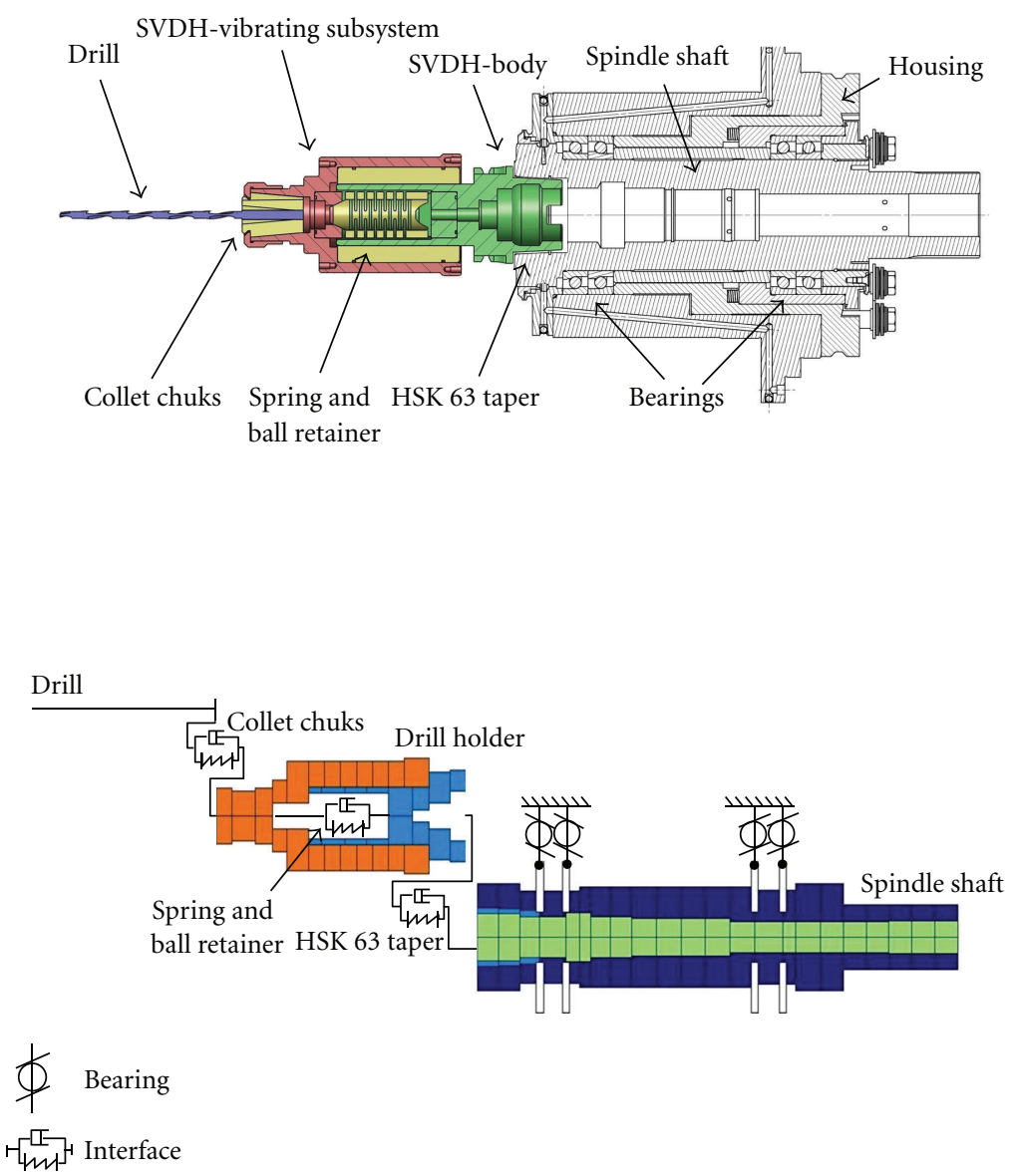

FIGURE 1: The spindle-SVDH-tool system and its finite element model.

behaviour parameters were integrated to obtain the assembled global model.

2.1. Modelling of Structural Subsystems. The spindle SVDH tool system is composed of four structural subsystems: the drill, the SVDH-vibrating subsystem, the SVDH body, and the spindle. The motion of the rotating structure is considered as the superposition of rigid and elastic body displacements. Dynamic equations were obtained using Lagrange formulation associated with a finite element method. Due to the size of the rotor sections, shear deformations had to be taken into account. Then, the rotating substructure was derived using Timoshenko beam theory. The relevant shape functions were cubic in order to avoid shear locking. A special three-dimensional rotor-beam element with two nodes and six degrees of freedom per node was developed in the corotational reference frame. The damping model used draws on Rayleigh viscous equivalent damping, which makes it possible to regard the damping matrix $\mathrm{D}$ as a linear combination of the mass matrix $\mathbf{M}$ and the spindle rigidity matrix K

$$
\mathbf{D}=a \mathbf{M}+b \mathbf{K}
$$

where $a$ and $b$ are damping coefficients.
The set of differential equations can be written as

$$
\mathbf{M}\left(\mathbf{q}_{N}\right) \ddot{\mathbf{q}}_{N}+\left(\mathbf{C}\left(\mathbf{q}_{N}, \dot{\mathbf{q}}_{N}\right)+\mathbf{D}\right) \dot{\mathbf{q}}_{N}+\mathbf{K} \mathbf{q}_{N}=\mathbf{F}(t),
$$

where $\mathbf{M}$ is the mass matrix, and $\mathbf{C}$ matrix contains the rotational dynamics effects. $\mathbf{q}_{N}$ and $\mathbf{F}(t)$ are the nodal displacement and force vectors. An accelerating rotor gives rise to previous time-variant equations (3), but treatment of the rotor using a pseudoconstant speed approach can still be described by means of linear time-invariant models and is valid in many cases (4):

$$
\mathbf{M}_{0} \ddot{\mathbf{q}}_{N}+(2 \Omega \mathbf{G}+\mathbf{D}) \dot{\mathbf{q}}_{N}+\left(\mathbf{K}-\mathbf{\Omega}^{2} \mathbf{N}\right) \mathbf{q}_{N}=\mathbf{F}(t),
$$

where $\mathbf{M}_{0}$ is the constant part of matrix $\mathbf{M}$, and $\mathbf{G}$ and $\mathbf{N}$ proceed from the decomposition of matrix $\mathbf{C}$.

2.2. Modelling Angular Contact Ball Bearings. The rotating system is supported by four (two front and two rear) hybrid angular contact bearings. The rolling bearing stiffness matrices were calculated using in-house software developed on the basis of Lim and Singh's [20] formulation. The bearing stiffness model represents the load-displacement relation combined with the Hertzian contact stress principle and was calculated around a static function point characterised by the bearing preload: $\delta$. Based on Rantatalo's prediction [9], 


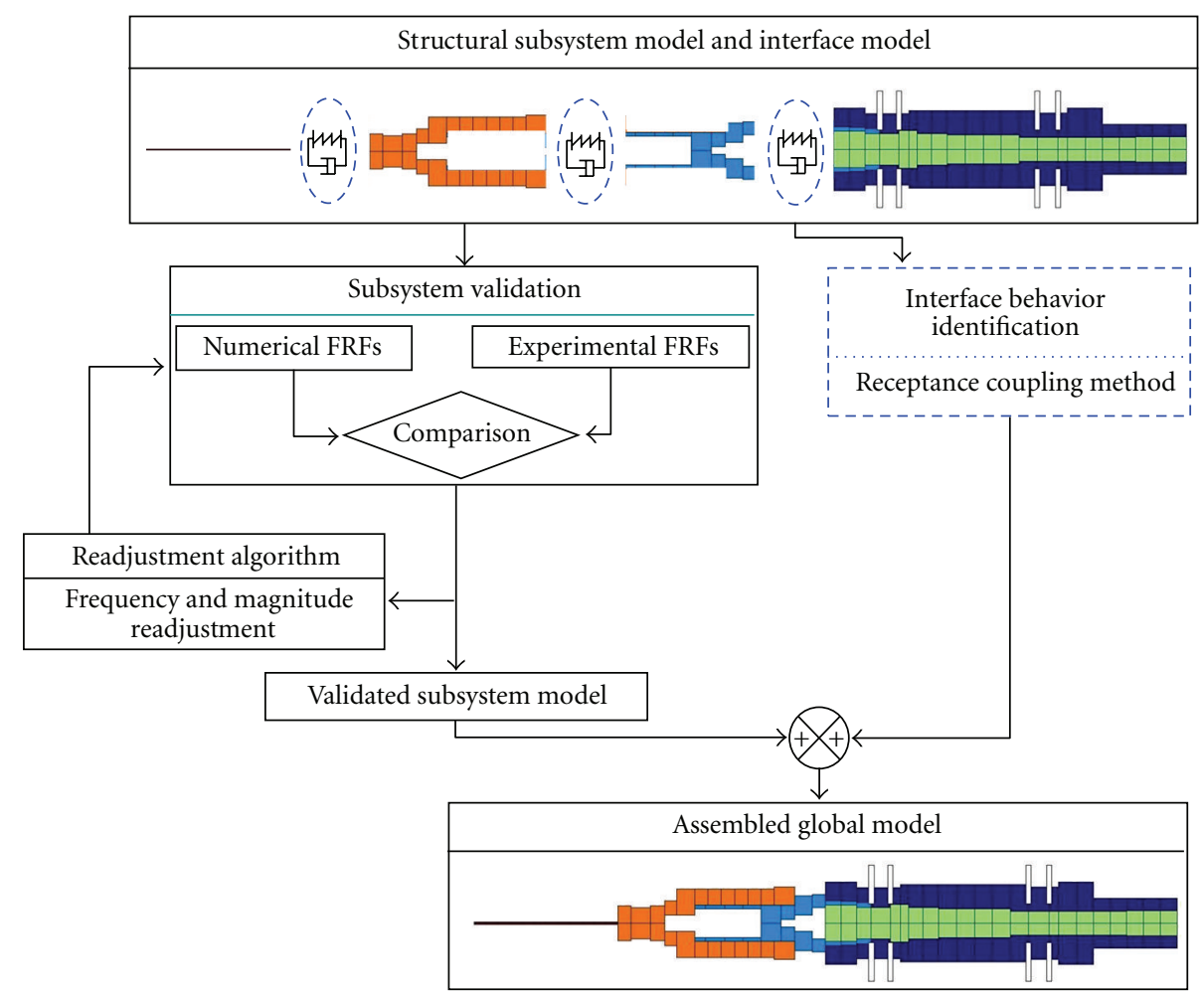

FIGURE 2: Development of the numerical model.

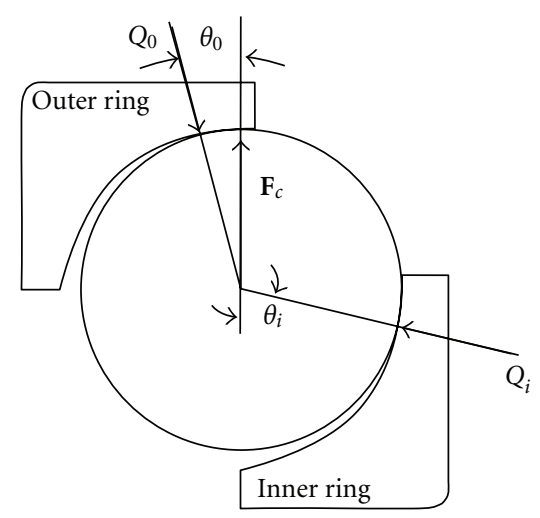

(a) Angular contact ball bearing Hertzian and centrifugal force

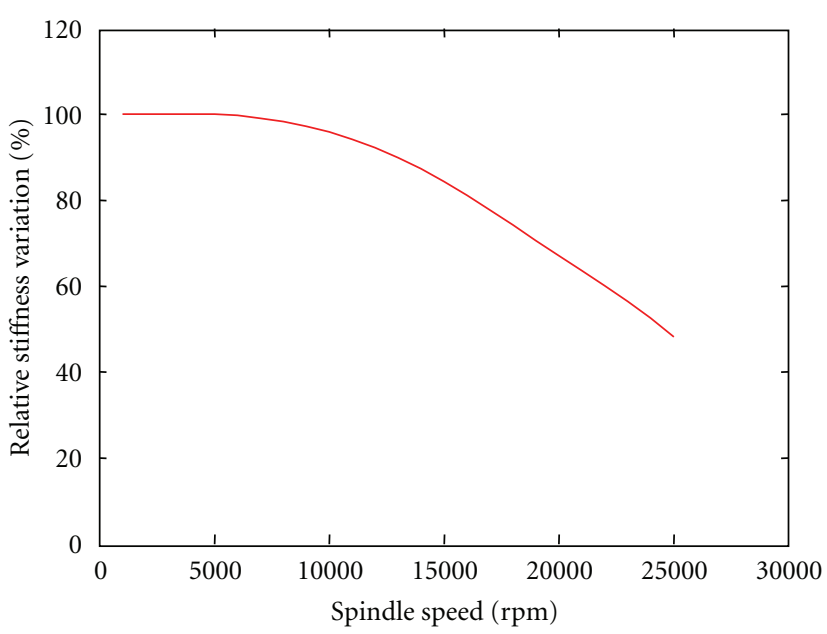

(b) Bearing stiffness variation

FIGURE 3: Bearing stiffness variation depending on spindle speed [12].

the initially calculated bearing stiffness is spindle speed dependent because of the gyroscopic and centrifugal force $\mathbf{F}_{c}$, which acts on each ball (Figure 3(a)). As the speed increased, the load conditions between the balls and the rings in the bearing changed because of the centrifugal force (Figure 3(a)). Then, speed-dependent bearing stiffness was integrated into the global spindle FEM and influenced the natural frequencies of the spindle-SVDH-tool unit under consideration.
2.3. Structural Systems Dynamic Readjustment. While detailed knowledge of the spindle-SVDH-tool system is in general not available in a manufacturing environment, models need to be readjusted in order to fit experimental results. The readjustment parameters are the Young modulus: $E$, the damping coefficients: $(a, b)$, and the rolling bearing preload: $\delta$. The readjustment procedure is proposed to tune the previous variable in order to fit the model results to the experimental frequency response function (FRF). These 


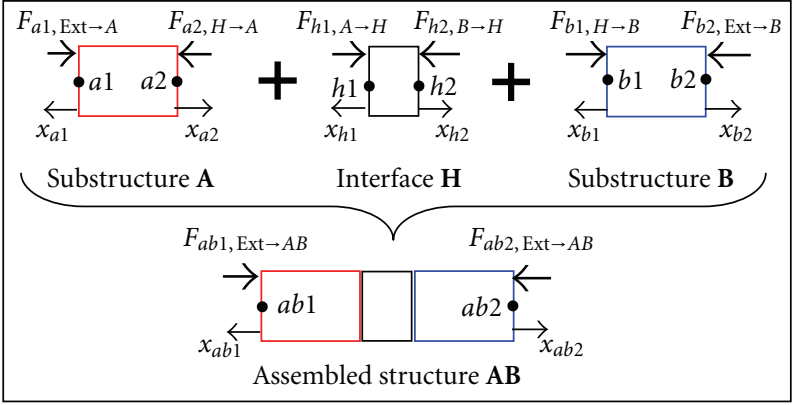

FIGURE 4: Receptance coupling notation.

parameters are readjusted by minimizing the gap between the measured and the modelled tool tip node FRF for nonrotating components, using an optimisation routine and a least squares type objective function defined as

$$
r=\sum_{\omega=\omega_{\min }}^{\omega_{\max }}\left(\mathbf{H}_{\mathrm{num}}(\omega,(E, a, b, \delta))-\mathbf{H}_{\exp }(\omega)\right)^{2},
$$

where $\mathbf{H}_{\text {num }}(\omega,(E, a, b, \delta))$ and $\mathbf{H}_{\exp }(\omega)$ are, respectively, the numerical and experimental FRF.

\subsection{Modelling and Identification of Spindle-SVDH-Tool Inter-} faces. The dynamic behaviour of the interfaces represented by the HSK63 taper, spring and ball retainer, and collet chuck was taken into account. The identification procedure of the interface models was based on the receptance coupling method.

2.4.1. Receptance Coupling Background. In this section, the RC equations are established. At the top of Figure 4, the substructure $\mathbf{A}$ and the substructure $\mathbf{B}$ are represented, connected by interface $\mathbf{H}$. At the bottom of Figure 4, the assembled structure is represented. This figure enables the excitation point and the measurement points used in the receptance coupling approach to be located.

The notation $\mathbf{A}_{\mathrm{ij}}(\omega)=\left.\left(x_{a i}(\omega) / F_{a j, \mathrm{Ext} \rightarrow A}(\omega)\right)\right|_{F_{a k, \mathrm{Ext} \rightarrow A=0, \forall k \neq j}}$ refers to the spatial receptance vector, whose output is the translation of point $a i$ and whose input is the force at point $a j$, when all other forces applied to substructure $\mathbf{A}$ are zero. The receptance of the assembled structure $\mathbf{A B}_{\mathrm{ij}}$ can be expressed according to the receptance of substructures $\mathbf{A}_{\mathrm{ij}}$ and $\mathbf{B}_{\mathbf{i j}}$ and the receptance of interface $\mathbf{H}$. The movement equations of each end point of substructures $\mathbf{A}$ and $\mathbf{B}$ and of structure $\mathbf{A B}$ are written as shown in (6) to (8). The behaviour of the interface, assuming zero mass, is given in (9), and the compatibility conditions are written in (10).

Substructure A:

$$
\begin{aligned}
& x_{a 1}=\mathbf{A}_{11} F_{a 1, \mathrm{Ext} \rightarrow A}+\mathbf{A}_{12} F_{a 2, H \rightarrow A}, \\
& x_{a 2}=\mathbf{A}_{21} F_{a 1, \mathrm{Ext} \rightarrow A}+\mathbf{A}_{22} F_{a 2, H \rightarrow A} .
\end{aligned}
$$

Substructure B:

$$
\begin{aligned}
& x_{b 1}=\mathbf{B}_{11} F_{b 1, H \rightarrow B}+\mathbf{B}_{12} F_{b 2, \text { Ext } \rightarrow B}, \\
& x_{b 2}=\mathbf{B}_{21} F_{b 1, H \rightarrow B}+\mathbf{B}_{22} F_{b 2, \text { Ext } \rightarrow B} .
\end{aligned}
$$

Substructure AB:

$$
\begin{aligned}
& x_{a b 1}=\mathbf{A B}_{11} F_{a b 1, \mathrm{Ext} \rightarrow A B}+\mathbf{A B}_{12} F_{a b 2, \mathrm{Ext} \rightarrow A B}, \\
& x_{a b 2}=\mathbf{A B}_{21} F_{a b 1, \mathrm{Ext} \rightarrow A B}+\mathbf{A B}_{\mathbf{2 2}} F_{a b 2, \mathrm{Ext} \rightarrow A B} .
\end{aligned}
$$

Interface:

$$
\begin{aligned}
x_{h 2}-x_{h 1} & =\mathbf{H} F_{h 2, B \rightarrow H}, \\
F_{h 2, B \rightarrow H} & =-F_{h 1, A \rightarrow H} .
\end{aligned}
$$

Compatibility conditions:

$$
\begin{gathered}
x_{a 1}=x_{a b 1}, \\
x_{b 2}=x_{a b 2}, \\
x_{h 1}=x_{a 2}, \\
x_{h 2}=x_{b 1}, \\
F_{a 2, H \rightarrow A}=F_{h 1, A \rightarrow H}, \\
F_{b 1, H \rightarrow \mathrm{B}}=F_{h 2, B \rightarrow H}, \\
F_{a 1, \mathrm{Ext} \rightarrow \mathrm{A}}=F_{a b 1, \mathrm{Ext} \rightarrow \mathrm{AB}}, \\
F_{b 2, \mathrm{Ext} \rightarrow B}=F_{a b 2, \mathrm{Ext} \rightarrow A B} .
\end{gathered}
$$

By integrating the compatibility and interface equations ((9) and (10)) into (6) to (8), system (11) is obtained

$$
\begin{gathered}
F_{a b 1, \mathrm{Ext} \rightarrow A B}\left(\mathbf{A}_{11}-\mathbf{A B}_{11}\right)+F_{a 2, H \rightarrow A}\left(\mathbf{A}_{12}\right) \\
+F_{a b 2, \mathrm{Ext} \rightarrow A B}\left(-\mathbf{A B}_{12}\right)=0, \\
F_{a b 1, \mathrm{Ext}} \rightarrow A B\left(\mathbf{A}_{21}\right)+F_{a 2, H \rightarrow A}\left(\mathbf{A}_{22}+\mathbf{B}_{11}\right) \\
+F_{a b 2, \mathrm{Ext} \rightarrow A B}\left(-\mathbf{B}_{12}\right)=0, \\
F_{a b 1, \mathrm{Ext} \rightarrow A B}\left(-\mathbf{A B}_{21}\right)+F_{a 2, H \rightarrow A}\left(-\mathbf{B}_{21}\right) \\
+F_{a b 2, \mathrm{Ext} \rightarrow A B}\left(\mathbf{B}_{22}-\mathbf{A B}_{22}\right)=0,
\end{gathered}
$$

$A B_{11}$ and $A B_{21}$ are elaborated from the definition of

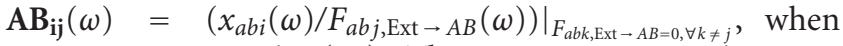
$F_{a b 2, \mathrm{Ext} \rightarrow A B}=0$ in (11). The receptance equations are obtained ((12)-(13))

$$
\begin{gathered}
\mathrm{AB}_{11}=\mathbf{A}_{11}-\mathbf{A}_{12}\left[\mathbf{B}_{11}+\mathbf{A}_{22}+\mathbf{H}\right]^{-1} \mathbf{A}_{21}, \\
\mathrm{AB}_{21}=\mathbf{A}_{21}\left[\mathbf{B}_{11}+\mathbf{A}_{22}+\mathbf{H}\right]^{-1} \mathbf{B}_{21} .
\end{gathered}
$$

Similarly, to obtain $\mathbf{A B}_{22}$ and, $\mathbf{A B}_{12}$ it is sufficient to impose $F_{a b 1, \mathrm{Ext} \rightarrow A B}=0$ in (11). Then the standard receptance equations are obtained(14)

$$
\begin{gathered}
\mathrm{AB}_{22}=\mathbf{B}_{22}-\mathbf{B}_{12}\left[\mathbf{B}_{11}+\mathbf{A}_{22}+\mathbf{H}\right]^{-1} \mathbf{B}_{21}, \\
\mathbf{A B}_{12}=\mathbf{A}_{12}\left[\mathbf{B}_{11}+\mathbf{A}_{22}+\mathbf{H}\right]^{-1} \mathbf{B}_{12} .
\end{gathered}
$$

From the receptance of the system substructures $\mathbf{A}_{\mathrm{ij}}, \mathbf{B}_{\mathrm{ij}}$, associated with the interface model $\mathbf{H}$, the reconstruction of the assembled structure FRF: $\mathbf{A B}_{\mathrm{ij}}$ is possible. 


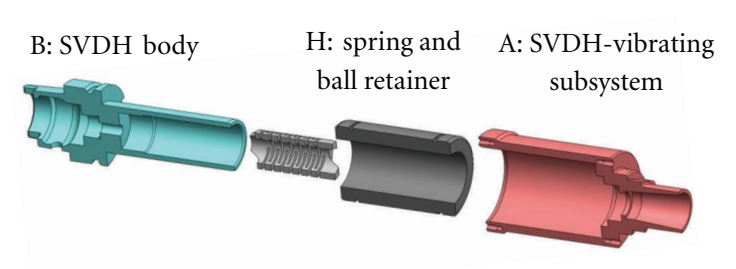

(a)

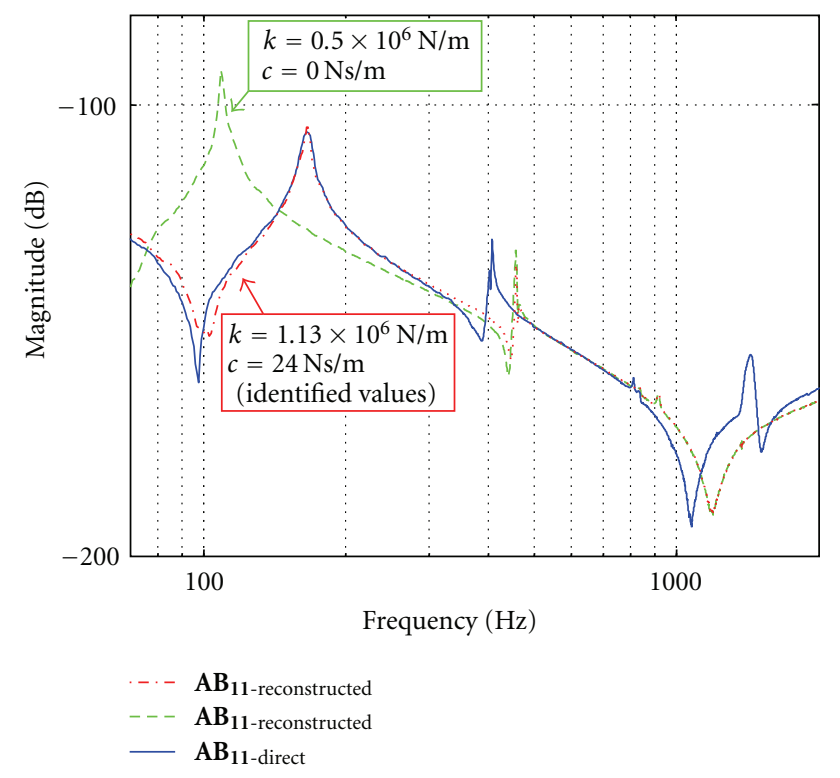

(b)

Figure 5: (a) Components (SVDH-body, SVDH vibrating subsystem) and interfaces (spring and ball retainer), (b) identification of the stiffness: $k$ and the damping factor: $c$ by minimizing the gap between $\mathbf{A B}_{11 \text {-reconstructed }}$ and $\mathrm{AB}_{11 \text {-direct }}$.

\subsubsection{Application to the SVDH-Vibrating Subsystem/SVDH} Body Interface. The interface between the SVDH-vibrating subsystem and the SVDH body is a prismatic joint whose axial stiffness is controlled by a spring. The SVDH-vibrating subsystem is guided in axial translation through a ball retainer, which controls the radial stiffness of the interface. Figure 5(a) shows these components. The axial dynamic behaviour of the interface was modelled using a spring damper: $\mathbf{H}_{\text {model }}=\mathbf{1} /(k+i c \omega)$. This interface was assumed to be rigid in the radial direction.

The $k$ and $c$ values were determined by minimizing the following $r$ criteria:

$$
r=\sum_{\omega=\omega_{0}-\varepsilon}^{\omega_{0}+\varepsilon}\left(\mathbf{A B}_{11-\text { reconstructed }}(\omega, k, c)-\mathbf{A B}_{11-\text { direct }}(\omega)\right)^{2},
$$

where $\mathbf{A B}_{11 \text {-reconstructed }}$ and $\mathbf{A B}_{11 \text {-direct }}$ represent, respectively, the RC-constructed FRF obtained by (12) and the measured FRF on the assembled system. $\omega_{0}$ is the interface mode pulsation. The optimisation procedure was carried out on a $3 \mathrm{~dB}$ bandwidth around $\omega_{0}$. In Figure 5(b), it can be noticed that the $\mathbf{A B}_{11-\text { reconstructed }}$ and $\mathbf{A} \mathbf{B}_{11-\text { direct }}$ curves are in good agreement, which enables the spring-damper interface model and the identified values $\left(k=1.13 \times 10^{6} \mathrm{~N} / \mathrm{m}\right.$ and $c=$ $24 \mathrm{Ns} / \mathrm{m}$ ) to be validated.

2.4.3. Application to the Collet Chuck Interface. The interface between the drill and the SVDH-vibrating subsystem is a collet chuck joint. Receptance $\mathbf{A B}_{21 \text {-direct }}$ is preferred to $\mathbf{A B}_{11-\text { direct }}$ in order to facilitate the experimental identification procedure. Moreover, the mass of the accelerometer is not negligible compared to the mass of the drill. In addition, the SVDH-vibrating subsystem is not in the same material configuration when the collet chuck is tightened on a drill as when it is not. For these reasons, receptance $\mathbf{A B}_{21 \text {-reconstructed }}$ (13) was obtained using numerical receptance for components $A$ and $B$. The identification procedure provided collet chuck stiffness and damping factors of, respectively, $14.8 \times$ $10^{6} \mathrm{~N} / \mathrm{m}$ and $4 \mathrm{Ns} / \mathrm{m}$.

2.4.4. Application to the HSK 63 Taper Interface. The experimental modal analyses carried out on the spindle/SVDH body system in the axial direction allow the HSK 63 interface to be considered as a rigid connection (Figure 6). Indeed, no specific mode for the HSK 63 taper interface appears between 0 and $3000 \mathrm{~Hz}$.

2.5. Model Assembly and Experimental Validation. As in a classic finite element procedure, dynamic equations of the overall system, composed of the drill, the SVDH-vibrating subsystems, the SVDH body, and the spindle, were obtained by assembling element matrices. The spring-damper connection parameters between the drill and the SVDH-vibrating subsystems and between the SVDH-vibrating subsystems and SVDH body, identified by the receptance coupling method, enabled the rotor-beam models of the components to be assembled.

The spindle-SVDH-tool assembled model was validated by comparison between numerical and experimental FRF, as shown in Figure 7.

Figure 7(a) represents experimental and numerical axial FRF of the assembled system. The $60 \mathrm{~Hz}$ and $4700 \mathrm{~Hz}$ modes are, respectively, due to the spring-ball retainer and collet chuck interfaces as mentioned in the previous subsystem identification procedure. Some parasitical experimental bending modes at $193 \mathrm{~Hz}, 1237 \mathrm{~Hz}$, and $3433 \mathrm{~Hz}$ are present in the experimental FRF.

Figure 7(b) represents experimental and numerical radial FRF of the assembled system. The 193, 1237, and $3433 \mathrm{~Hz}$ modes are related to the drill's bending modes. The $376 \mathrm{~Hz}$ mode is controlled by the bearings. Additional numerical modes are present at 1830 and $3740 \mathrm{~Hz}$. These frequency peaks are related to the dynamics behaviour of the rear side of the spindle. They do not appear in the experimental FRF since the displacements of the rear side of the real spindle are blocked by the motor. For the axial and radial FRF, a good correspondence between the numerical and experimental curves enabled the numerical model to be used for further investigations. 


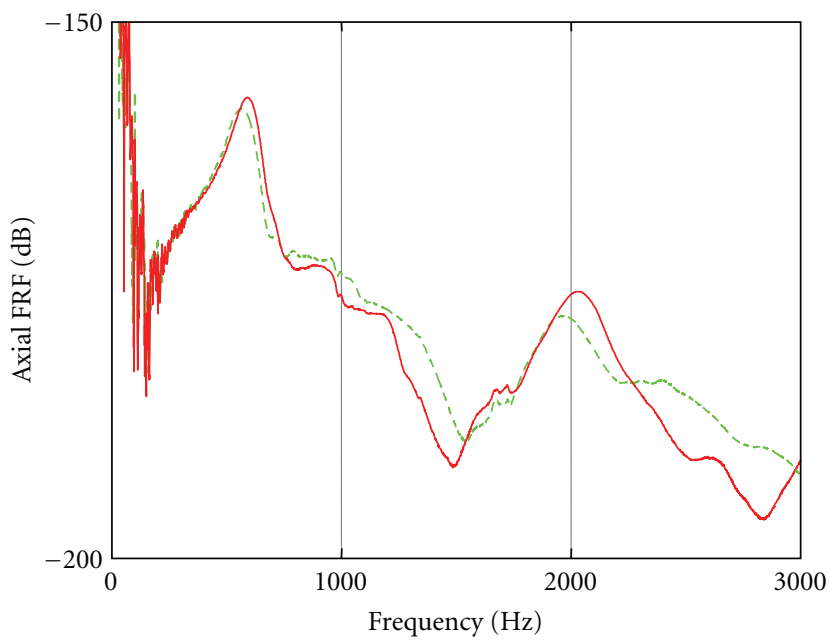

(a)

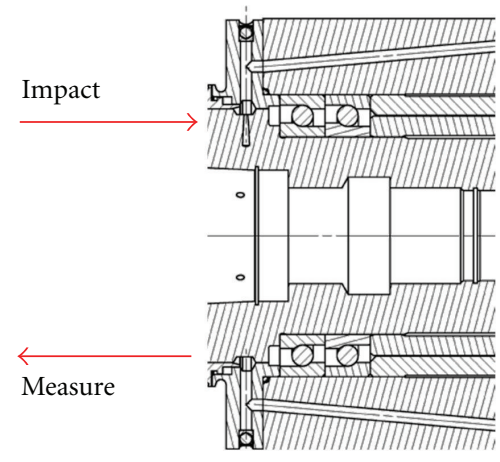

(b)

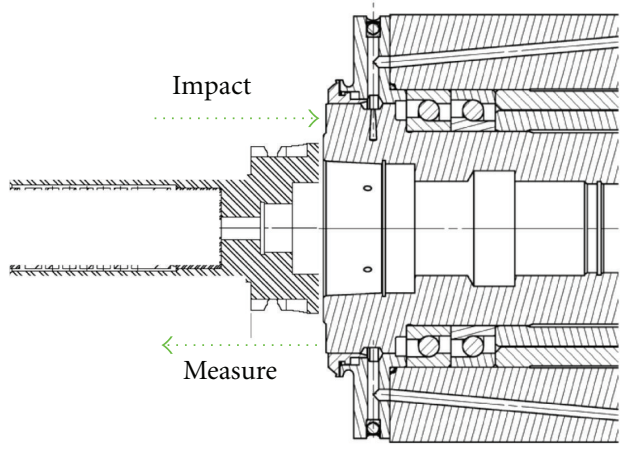

(c)

FIGURE 6: HSK 63 experimental modal analyses and associated axial FRF.

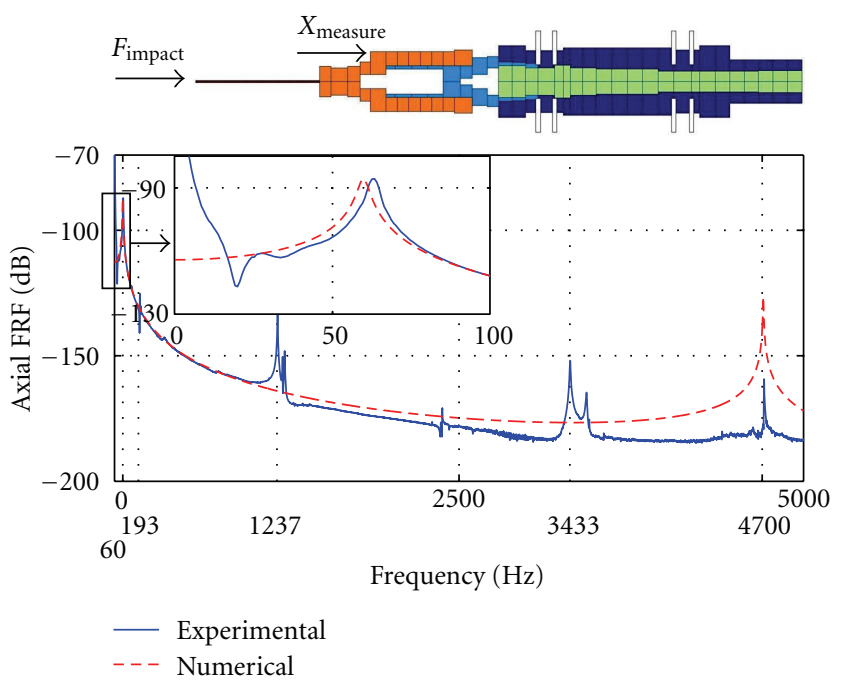

(a)
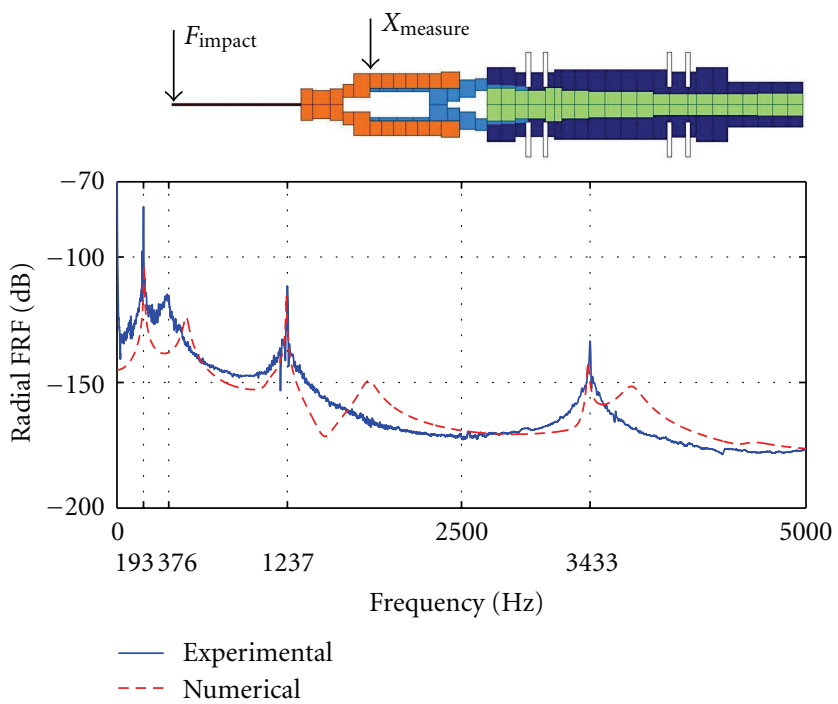

(b)

FIGURE 7: Numerical versus experimental system FRF. 


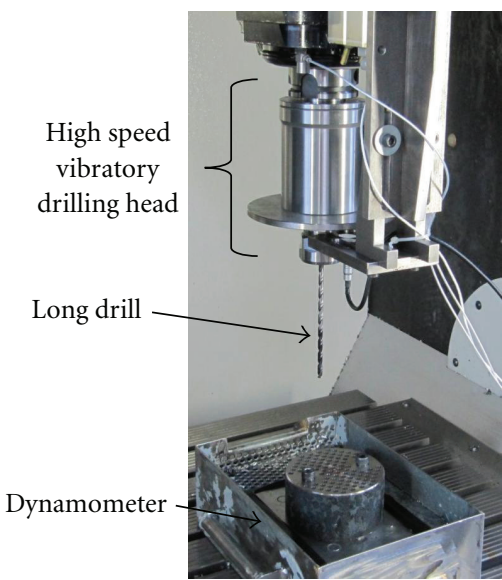

(a)

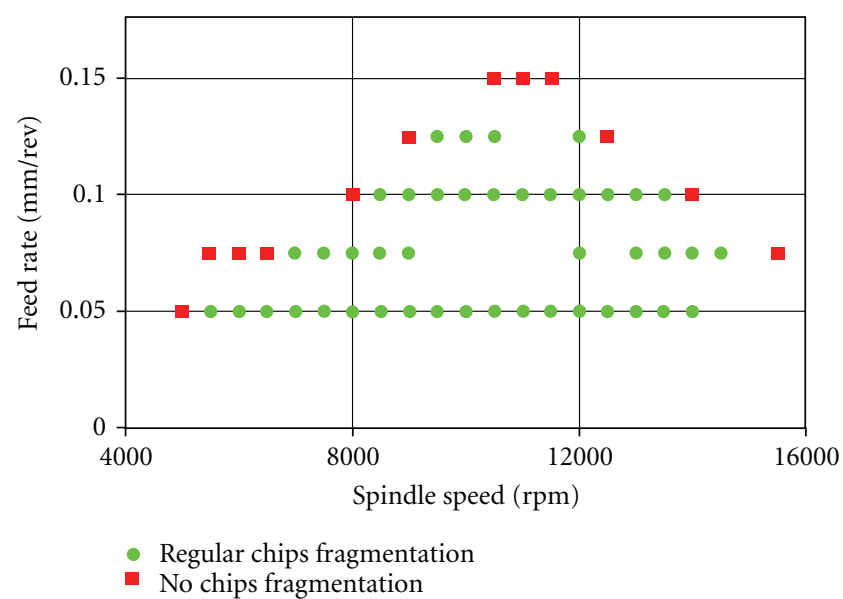

(b)

FIGURE 8: Experimental setup.

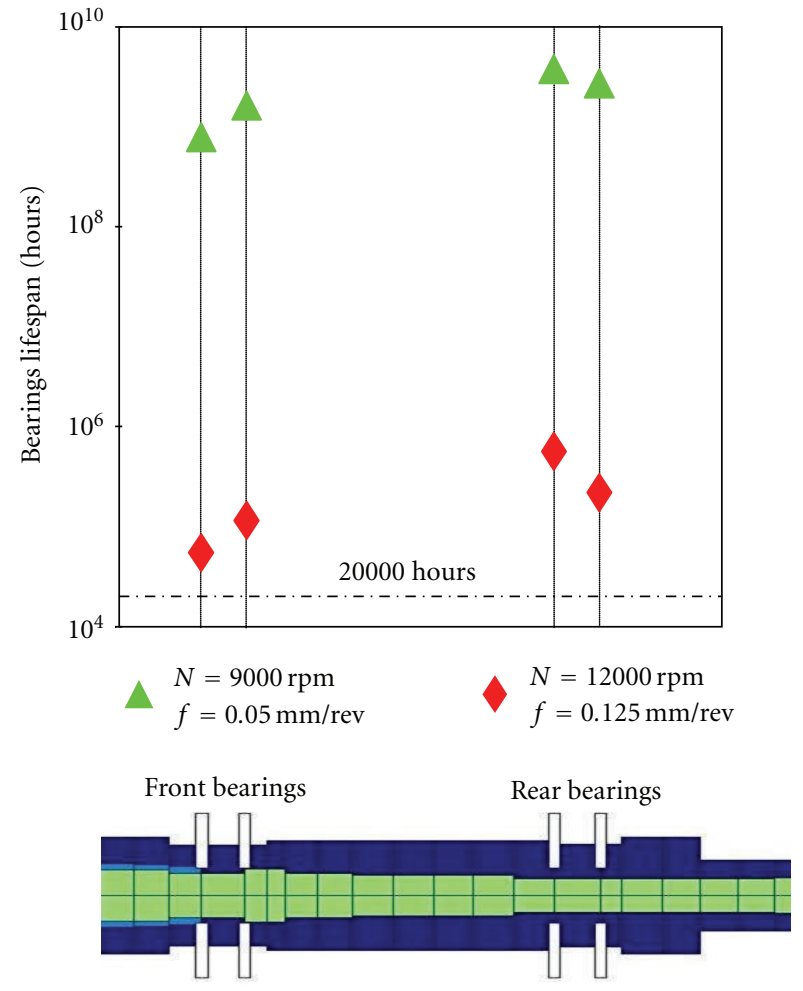

FIGURE 9: Influence of location and cutting conditions on bearings lifespan.

\section{Bearing Lifespan Predictions}

The industrial context of the proposed paper is to realize high-speed vibratory drilling operation, with a drill diameter of $5 \mathrm{~mm}$, with a drill depth of $100 \mathrm{~mm}$, without retreat cycles or lubrication, in a mass production system.

In this section, the numerical model will be used to predict bearings lifespan for various cutting conditions, in this context. The calculated bearing lifespan, thanks to experimental data, was compared to industrial recommendations in order to give rules of uses to obtain the maximum removal rate with respect to system lifespan.

For bearing lifespan calculations, experiments were carried out to measure the cutting force for different cutting conditions, using a three-component dynamometer (Kistler dynamometer type 9257B). High-speed vibratory drilling operations, representative of the industrial context, were performed on $35 \mathrm{MnV} 7$ steel, with a drill of $116 \mathrm{~mm}$ length and of $5 \mathrm{~mm}$ diameter. The experimental setup is represented in Figure 8(a). Rotation speed and feed rate were tested, respectively, between 5500 and $15500 \mathrm{rpm}$ and between 0.05 and $0.15 \mathrm{~mm} / \mathrm{rev}$. Only the drilling operations that led to regular chips fragmentation were used to predict bearing lifespan, as shown in Figure 8(b). The time-variant value of the radial forces, which depends on the angular orientation of the cutter as it rotates through the cut, was expanded into a Fourier series and then truncated to include only the fundamental frequency. These forces, combined with the numerical FRF of the model, between the excitation node and the bearings nodes, allow the resulting rolling bearings solicitation to be determined. The rolling bearings lifespan is

$$
L_{h}=\frac{10^{6}}{60 N}\left(\frac{C}{P}\right)^{3},
$$

where $C$ is the basic dynamic load and $P$ the equivalent dynamic load in Newtons. $N$ is the spindle speed in rpm. The values obtained are expressed in hours and can be compared to an industrial objective of 20000 hours.

3.1. Effect of Cutting Conditions on Bearing Lifespan. First, the bearings with the shortest lifespan is sought, because it is the element upon which the lifespan of the entire spindle depends. For all cutting conditions, the bearings with the shortest lifespan is always the front bearings. From Figure 9, which represents the extreme lifespan of the different bearings, the strong influence of the cutting conditions on the bearings lifespan can be noticed. Industrial recommendations for spindle lifespan are 20000 hours. The shortest calculated lifespan is 55000 and corresponds to the cutting 


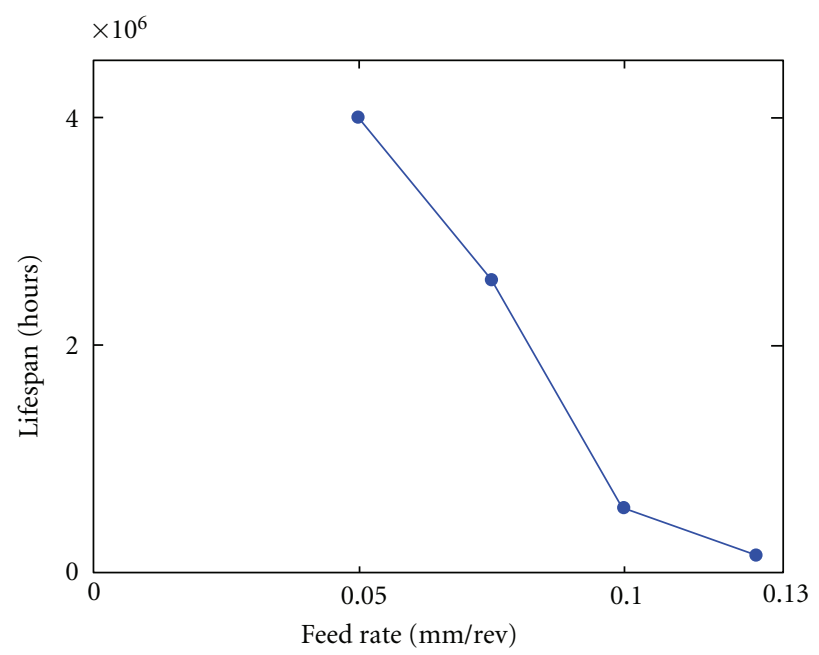

(a)

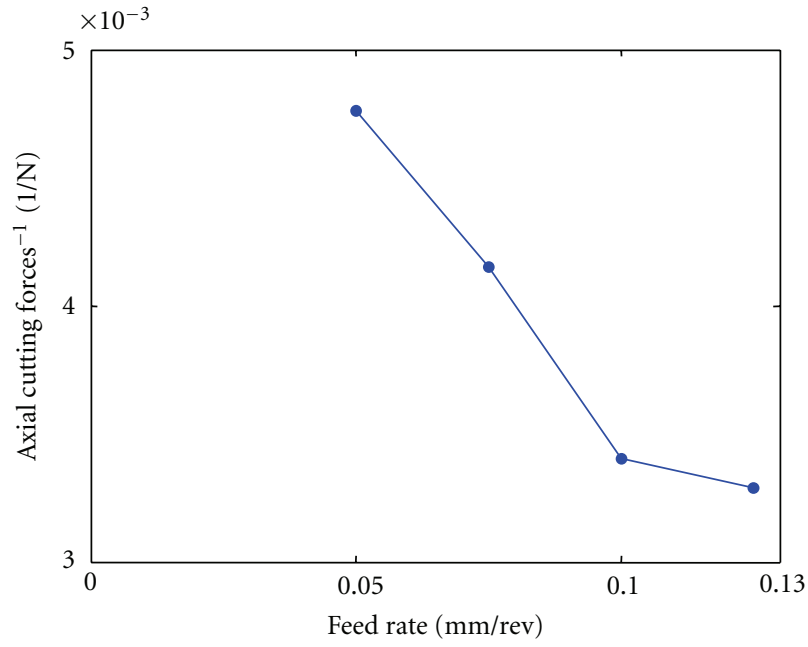

(b)

FIGURE 10: (a) Influence of feed rate on bearings lifespan, for a spindle speed of $12000 \mathrm{rpm}$, and (b) influence of the feed rate on the inverse of experimental axial cutting force magnitude.

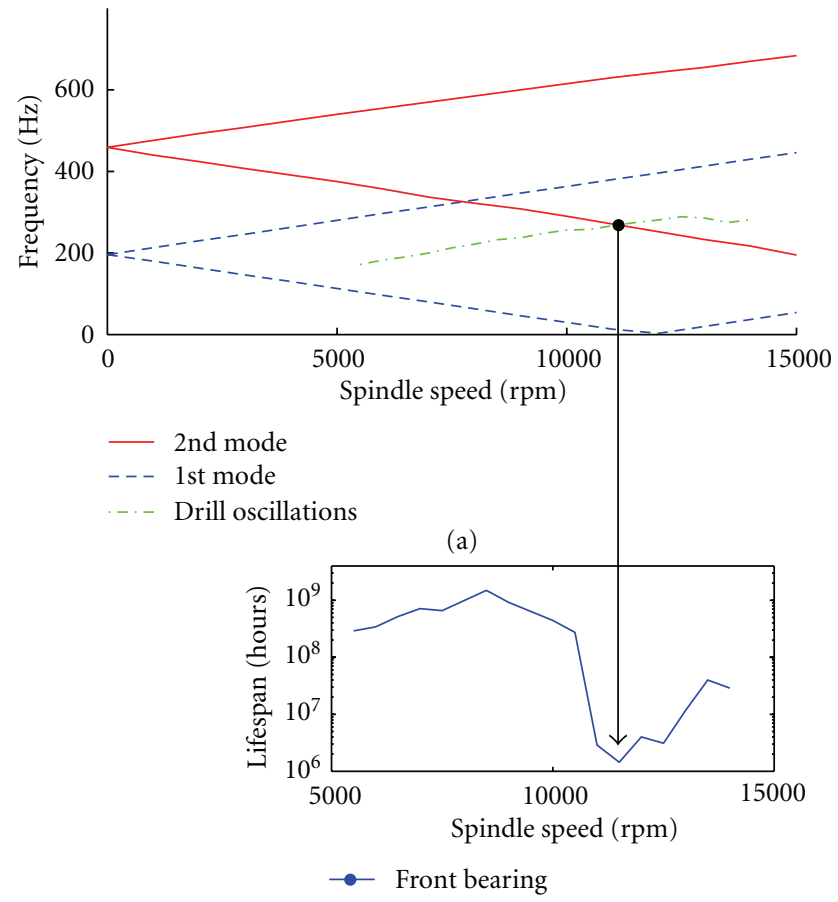

(b)

Figure 11: (a) Campbell diagram. (b) Influence of spindle speed on bearings lifespan, for a feed rate of $0.05 \mathrm{~mm} / \mathrm{rev}$.

conditions given rise to the best material removal rate $(N=$ $12000 \mathrm{rpm}, f=0.125 \mathrm{~mm} / \mathrm{rev})$. Thus, a high-speed vibratory drilling operation is always compatible with industrial standards, even under the best material removal rate cutting conditions.

Figure 10(a) shows the influence of the feed rate on bearings lifespan, for a spindle speed of $12000 \mathrm{rpm}$. Only feed rates higher than $0.05 \mathrm{~mm} / \mathrm{rev}$ were tested, because for feed rates below this limit, the productivity is too low and highspeed vibratory drilling loses its relevance. For feed rates higher than $0.125 \mathrm{~mm} / \mathrm{rev}$, no stable vibrations of the drill were obtained. The bigger the feed rate, the lower the bearings Lifespan. However, even the shortest bearings Lifespan is compatible with industrial standard. Hence, the optimal feed rate of $0.125 \mathrm{~mm} / \mathrm{rev}$ is retained. Figure 10 (b) shows that cutting forces are modified by the feed rate. Similarities between the curves in Figures 10(a) and 10(b) indicate that the bearing lifespan is mainly influenced by the feed rate.

Figure 11(b) shows the variations in bearing lifespan depending on spindle speed, for a feed rate of $0.05 \mathrm{~mm}$ per revolution. The curve enables the determination of a spindle speed at $8500 \mathrm{rpm}$ which maximizes bearing Lifespan and a spindle speed at $11500 \mathrm{rpm}$ which minimizes it. In order to optimize productivity, as shown on Figure 8(b), the recommended cutting spindle speed is $12000 \mathrm{rpm}$.

The curves of Figure 11 illustrate the dynamic effects due to high rotational speed, such as gyroscopic coupling and spin softening, on system behaviour, and hence on bearing lifespan. Figure 11(a) represents the effect of spindle speed on the first two radial modes of the system. The dotted line indicates the drill oscillation frequency and is plotted from experimental data measured during high-speed vibratory drilling operations. The critical speed, at $11500 \mathrm{rpm}$, corresponds to the intersection of the radial mode frequencies with the excitation line due to the drill oscillations.

\section{Conclusions}

In this paper, a comprehensive approach to developing a hybrid model of the dynamic behavior of the spindle self-vibratory drilling head-tool system has been proposed. This approach has resulted in a numerical model enriched with physical data. The various components of the system are modelled using a specific beam element, taking into account the gyroscopic effects, centrifugal forces, and shear deflection. The receptance coupling method is used to identify the dynamic behavior of the interface. The complete system is 
then obtained by assembling the beam model of each component using spring-damper elements. Finally, the assembled model is validated by comparisons between numerical and experimental FRFs. The model is used to predict the influence of a high-speed vibratory drilling operation on the bearing lifespan. The predictions of bearing lifespan are used to give rules of uses of the high-speed vibratory drilling head.

\section{Nomenclature}

$\begin{array}{ll}\mathbf{A}_{\mathbf{i j}}: & \text { receptance vector of system } A \\ x_{a i}: & \text { translation of point } i \text { of system } A \\ F_{a j, \text { Ext } \rightarrow A}: & \text { force applied to system } A \text { at point } j \\ \mathbf{H}_{\text {model }}: & \text { interface model receptance } \\ k: & \text { interface stiffness } \\ c: & \text { interface damping factor } \\ \mathbf{A B}_{\mathrm{ij} \text {-reconstructed }}: & \text { system AB receptance, reconstructed } \\ & \text { using receptance-coupling equations } \\ \mathrm{AB}_{\mathbf{i j}-\text { direct }}: & \text { system AB receptance, directly } \\ \mathrm{D}: & \text { measured } \\ a, b: & \text { damping matrix } \\ \mathbf{M}: & \text { damping coefficients } \\ \mathbf{K}: & \text { mass matrix } \\ \mathbf{C :} & \text { stiffness matrix } \\ \mathbf{G}: & \text { rotational dynamic effects matrix } \\ \mathbf{N}: & \text { gyroscopic matrix } \\ \Omega: & \text { spin softening effects matrix } \\ \mathbf{q}_{N}: & \text { rotor angular velocity } \\ \mathbf{F}: & \text { nodal displacement } \\ E: & \text { force vector } \\ \delta: & \text { Young modulus } \\ N: & \text { rolling bearing preload } \\ L_{h}: & \text { spindle speed in rpm } \\ C: & \text { bearing lifespan } \\ P: & \text { basic dynamic load } \\ & \text { equivalent dynamic bearing load. }\end{array}$

\section{References}

[1] A. M. Gouskov, S. A. Voronov, and S. A. Batzer, "Chatter synchronization in vibratory drilling," in Proceedings of the American Society of Mechanical Engineers, vol. 68, pp. 263-270, 2000.

[2] S. Tichkiewitch, G. F. Moraru, D. Brun-Picard, and A. Gouskov, "Self-excited vibration drilling models and experiments," CIRP Annals, vol. 51, no. 1, pp. 311-314, 2002.

[3] N. Guibert, H. Paris, and J. Rech, "A numerical simulator to predict the dynamical behavior of the self-vibratory drilling head," International Journal of Machine Tools and Manufacture, vol. 48, no. 6, pp. 644-655, 2008.

[4] J. Agapiou and C. Rivin, "Toolholder/spindle interfaces for CNC machine tools," CIRP Annals, vol. 44, no. 1, pp. 383-387, 1995.

[5] S. Smith, W. R. Winfough, and J. Halley, "The effect of drawbar force on metal removal rate in milling," CIRP Annals, vol. 48, no. 1, pp. 293-296, 1998.

[6] S. Jiang and S. Zheng, "A modeling approach for analysis and improvement of spindle-drawbar-bearing assembly dynamics," International Journal of Machine Tools and Manufacture, vol. 50, no. 1, pp. 131-142, 2010.
[7] A. Erturk, H. N. Ozguven, and E. Budak, "Effect analysis of bearing and interface dynamics on tool point FRF for chatter stability in machine tools by using a new analytical model for spindle-tool assemblies," International Journal of Machine Tools and Manufacture, vol. 47, no. 1, pp. 23-32, 2007.

[8] B. R. Jorgensen and Y. C. Shin, "Dynamics of spindle-bearing systems at high speeds including cutting load effects," Journal of Manufacturing Science and Engineering, vol. 120, no. 2, pp. 387-394, 1998.

[9] M. Rantatalo, J. O. Aidanpaa, B. Goransson, and P. Norman, "Milling machine spindle analysis using FEM and non-contact spindle excitation and response measurement," International Journal of Machine Tools and Manufacture, vol. 47, no. 7-8, pp. 1034-1045, 2007.

[10] H. Li and Y. C. Shin, "Integrated dynamic thermo-mechanical modeling of high speed spindles, part 1: model development," Journal of Manufacturing Science and Engineering, vol. 126, no. 1, pp. 148-158, 2004.

[11] Y. Cao and Y. Altintas, "Modeling of spindle-bearing and machine tool systems for virtual simulation of milling operations," International Journal of Machine Tools and Manufacture, vol. 47, no. 9, pp. 1342-1350, 2007.

[12] V. Gagnol, C. B. Bouzgarrou, P. Ray, and C. Barra, "Modelbased chatter stability prediction for high-speed spindles," International Journal of Machine Tools and Manufacture, vol. 47, no. 7-8, pp. 1176-1186, 2007.

[13] I. Mane, V. Gagnol, B. C. Bouzgarrou, P. Ray, and C. Berra, "Stability-based spindle speed control during flexible workpiece high-speed milling," International Journal of Machine Tools and Manufacture, vol. 48, no. 2, pp. 184-194, 2008.

[14] A. Erturk, H. N. Ozguven, and E. Budak, "Analytical modeling of spindle-tool dynamics on machine tools using Timoshenko beam model and receptance coupling for the prediction of tool point FRF," International Journal of Machine Tools and Manufacture, vol. 46, no. 15, pp. 1901-1912, 2006.

[15] T. L. Schmitz, K. Powell, D. Won, G. S. Duncan, W. G. Sawyer, and J. C. Ziegert, "Shrink fit tool holder connection stiffness/damping modeling for frequency response prediction in milling," International Journal of Machine Tools and Manufacture, vol. 47, no. 9, pp. 1368-1380, 2007.

[16] H. Ahmadian and M. Nourmohammadi, "Tool point dynamics prediction by a three-component model utilizing distributed joint interfaces," International Journal of Machine Tools and Manufacture, vol. 50, no. 11, pp. 998-1005, 2010.

[17] Y. Ren and C. F. Beards, "Identification of joint properties of a structure using FRF data," Journal of Sound and Vibration, vol. 186, no. 4, pp. 567-587, 1995.

[18] M. Movahhedy and J. Gerami, "Prediction of spindle dynamics in milling by sub-structure coupling," International Journal of Machine Tools and Manufacture, vol. 46, no. 3-4, pp. 243$251,2006$.

[19] O. Ozsahin, A. Erturk, H. N. Ozguven, and E. Budak, "A closed-form approach for identification of dynamical contact parameters in spindle-holder-tool assemblies," International Journal of Machine Tools and Manufacture, vol. 49, no. 1, pp. 25-35, 2009.

[20] T. C. Lim and R. Singh, "Vibration transmission through rolling element bearings, part I to part III," Journal of Sound and Vibration, vol. 139, no. 2, pp. 179-199, 201-225, 1990. 

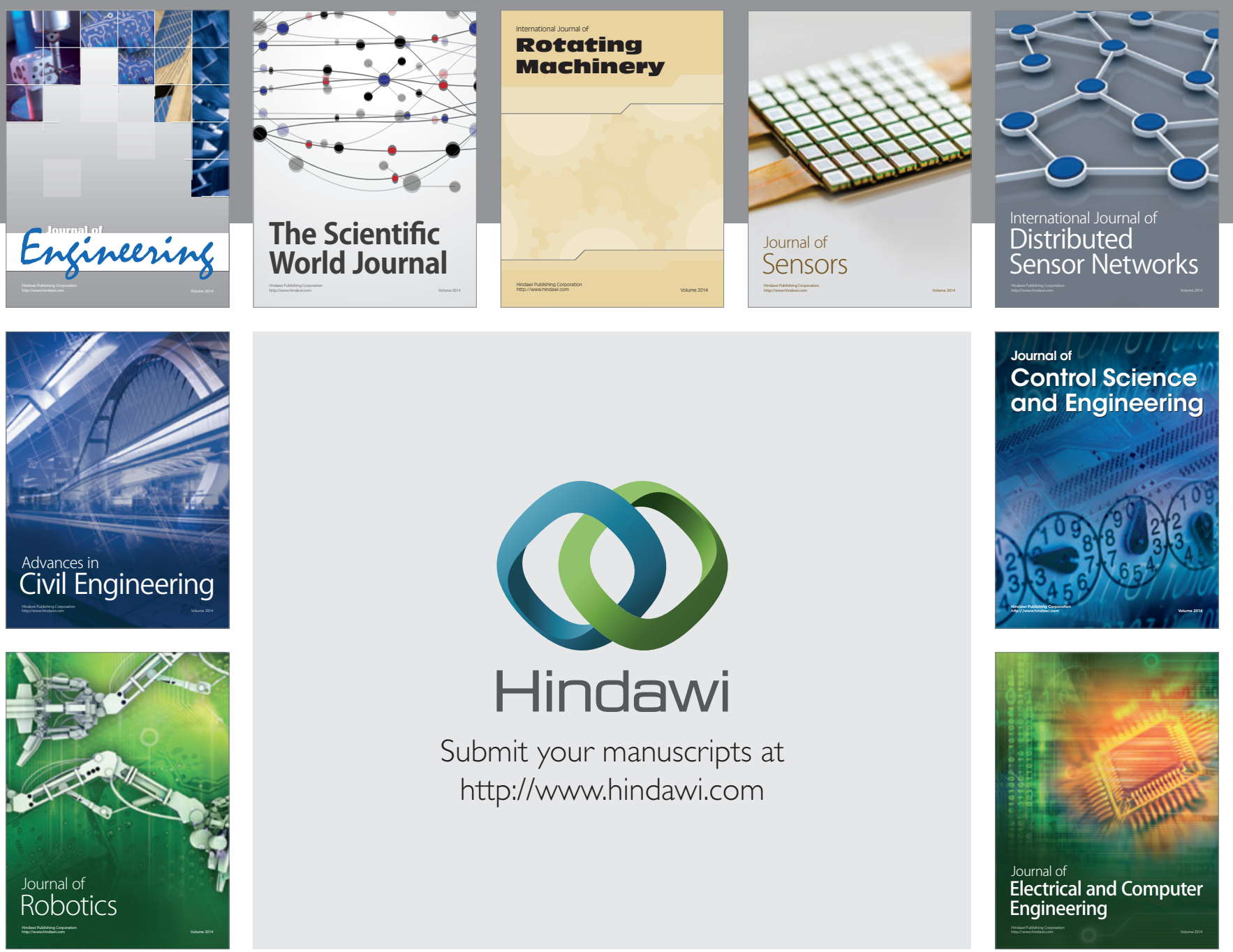

Submit your manuscripts at

http://www.hindawi.com
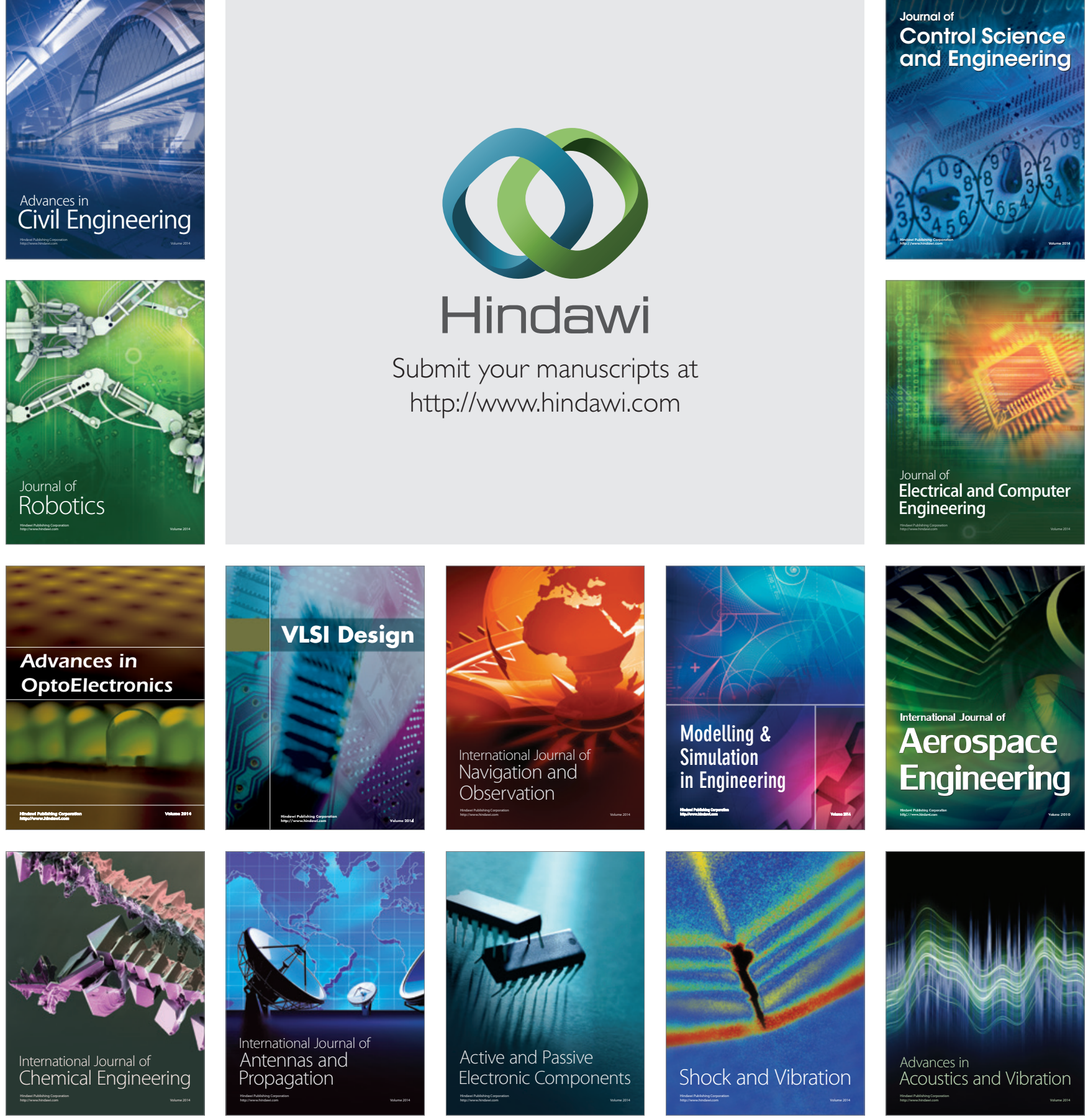